\title{
Predicting Pain in Total and Partial Knee Arthroplasty
}

\author{
Tatsuya Sueyoshi',2, Wesley G. Lackey ${ }^{1,2}$, Robert A. Malinzak ${ }^{1,2}$, John B. Meding1,2, \\ Alex Farris ${ }^{1,2}$, Kenneth E. Davis ${ }^{1,2}$, Merrill A. Ritter ${ }^{1,2}$ \\ ${ }^{1}$ Indiana Research Foundation (JRSI), Center for Hip and Knee Surgery, Mooresville, USA \\ ${ }^{2}$ Midwest Center for Joint Replacement, Indianapolis, USA \\ Email: jrsiresearch1@gmail.com
}

Received 30 April 2015; accepted 15 June 2015; published 18 June 2015

Copyright (C) 2015 by authors and Scientific Research Publishing Inc.

This work is licensed under the Creative Commons Attribution-NonCommercial International License (CC

BY-NC).

http://creativecommons.org/licenses/by-nc/4.0/

(c) (i) @ Open Access

\section{Abstract}

Object: Although pain relief is a primary goal of a total knee arthroplasty (TKA) and partial knee arthroplasty (PKA), a significant number of arthroplasty patients experience unexplained pain. This study attempts to determine preoperative or intraoperative factors that may predict pain after knee arthroplasty. Methods: 2585 primary TKAs and 423 PKAs were performed between 1993 and 2013. Infections, loosening, and revision arthroplasty were excluded. Knee Society scores, demographics, component sizes, pre- and postoperative alignment, treatment of the posterior cruciate ligament, range of motion, and diagnosis were analyzed. Statistical analysis utilized repeated measures ANOVA. Results: Significant predictors of increased pain after TKA were preoperative varus alignment $>5^{\circ}(\mathrm{p}=0.0042)$, postoperative flexion range of motion $<105^{\circ}(\mathrm{p}<$ 0.0001), postoperative flexion contracture $>5^{\circ}(p=0.0006)$, small tibial component sizes $(p=$ $0.0080)$, excision of the posterior cruciate ligament $(p=0.0259)$, and diagnosis as osteonecrosis $(p=0.0077)$. Factors not associated with pain included age, body mass index, gender, postoperative alignment and bone quality. For PKA, age was the only factor associated with pain. Conclusions: Six factors were found to be relevant to postoperative pain in TKA. For PKA, reported pain is not associated with any of the same factors, but is associated with age. Surgeons should be aware of these risk factors as we continue to understand pain after total and partial knee arthroplasty.

\section{Keywords}

Postoperative Pain, Total Knee Arthroplasty, Partial Knee Arthroplasty, Alignment, Tibial Size 


\section{Introduction}

Pain following a total knee arthroplasty (TKA) continues in a small but significant number of dissatisfied patients, with $14 \%$ of patients unsatisfied with their arthroplasty in one study [1]. In many cases, the cause of this pain is not immediately apparent, though it may arise from patient factors found before or after the arthroplasty. Few studies, to our knowledge, focus solely on examining the patient factors in TKA that associate with pain, as uncovered by a systematic review [2] that found inconsistent influence of patient characteristics among 64 analyzable studies. Patients with greater preoperative pain are reported to have more postoperative pain, use more home therapy, and more often require manipulation under anesthesia [3]. Preoperative depression and anxiety were associated with increase in pain reporting [3].

The present study examines both preoperative and postoperative factors associated with otherwise unexplained pain. Our hypothesis was that this pain should be associated with certain underlying clinical or demographic variables.

\section{Methods}

Our TKA study group consisted of 2585 primary anatomic graduated component (AGC, Biomet, Warsaw, IN) cruciate-retaining TKAs implanted into 1803 patients. The procedures were performed by six surgeons between June 1993 and August 2006, using similar surgical techniques, instrumentation, and postoperative rehabilitation programs. The surgeries had complete preoperative, intraoperative, and postoperative data regarding alignment, pain, range of motion, state of the posterior cruciate ligament, diagnosis, and component sizes. An additional cohort of $n=423$ partial knees (Oxford Knee System, Biomet, Warsaw, IN) between February 2002 and March 2013 implanted in 310 patients were analyzed for similarities and differences in those covariates that affected postoperative pain.

Patients were evaluated pre- and post-operatively using the objective and functional scales of the Knee Society clinical rating system at 2 months, 6 months, and 1, 3, 5, 7, 10, and 12 years, as available. Pain scores were obtained for each knee and analyzed for the present study, from 0 (severe pain) to 50 (no pain). Each knee had at least one follow-up between 2 and 8 years after primary arthroplasty, which has been shown to be the most stable time range for postoperative pain scores [4]. We examined data in this follow-up range as well as demographic and preoperative variables for associations with decreased Knee Society pain scores.

The three significant ranged variables (preoperative varus, flexion, and flexion contracture) were divided into two groups in order to find quantitative differences within each variable. Preoperative alignment scores were divided into less than or equal to 5 degrees of varus and greater than 5 degrees of varus; flexion into greater than or equal to 105 degrees and less than 105 degrees [5]; and flexion contracture into less than 6 degrees and greater than or equal to 6 degrees [6]. The differences among the four available tibial tray sizes $(65 \mathrm{~mm}, 70 \mathrm{~mm}, 80$ $\mathrm{mm}$, and $85 \mathrm{~mm}$ ) were also examined.

Statistical analysis was performed by repeated measures ANOVA on the 3, 5, and 7-year follow-ups using the compound symmetry covariance matrix design. The estimation technique was the minimum variance quadratic unbiased method. The repeated subject was each knee joint. Preoperative, intraoperative, and postoperative variables (as listed as results) were examined for their relation to pain. Significance for each variable was set at $\mathrm{p}<$ 0.05 .

\section{Results}

There were 1121 female (62.2\%) and 682 male (37.8\%) patients in the TKA cohort. Diagnoses included osteoarthritis (OA, 2550, 98.6\%), rheumatoid arthritis (RA, 26, 1.0\%), and osteonecrosis (ON, 9, 0.4\%). Average was $68.7 \pm 8.5$ years (range, 38 - 90 years). Average follow-up was $6.8 \pm 3.1$ years (range, 2.0 - 15.2 years).

Significant associations with pain were found in preoperative varus deformity $(\mathrm{p}=0.0106)$, limited postoperative flexion ( $p<0.0001)$, postoperative flexion contracture $(p=0.0005)$, smaller tibial tray size $(p=0.0026)$, diagnosis of osteonecrosis $(\mathrm{p}=0.0077)$, excision of the posterior cruciate ligament in a cruciate-retaining prosthesis $(p=0.0259)$, and bilateral surgery $(p=0.0056)$ (effect sizes shown in Table 1$)$. Significant associations were not found between pain and age ( $p=0.0737)$, preoperative flexion contracture $(p=0.0859)$, postoperative overall alignment $(p=0.0955)$, postoperative varus $(p=0.7627)$, postoperative valgus $(p=0.5391)$, femoral bone quality $(p=0.1587)$, gender $(p=0.3771)$, height $(p=0.5979)$, recession of the posterior cruciate ligament 
Table 1. Factors associated with pain scores in TKA.

\begin{tabular}{ccc}
\hline Factor & Difference & p-value of factor \\
\hline Preoperative varus $>5$ degrees & +0.8 points & $\mathrm{p}=0.0096$ \\
Flexion $<105$ degrees & -2.3 points & $\mathrm{p}<0.0001$ \\
Flexion contracture $>6$ degrees & -2.4 points & $\mathrm{p}=0.0006$ \\
PCL excision & -3.1 points & $\mathrm{p}=0.0259$ \\
Tibial size $($ vs. $85 \mathrm{~mm})$ & & $\mathrm{p}=0.0080$ \\
$65 \mathrm{~mm}$ & -1.0 points & $\mathrm{p}=0.0222$ \\
$70 \mathrm{~mm}$ & -0.8 points & $\mathrm{p}=0.0223$ \\
$80 \mathrm{~mm}$ & -0.3 points & $\mathrm{p}=0.4890$ \\
Osteonecrosis & -4.9 points & $\mathrm{p}=0.0077$ \\
\hline
\end{tabular}

( $p=0.6139)$, tibial bone quality $(p=0.6799)$, patient weight $(p=0.7620)$, and body mass index $(p=0.8533)$.

At an endpoint after 2 years, 5.2\% of knees (132 of 2571) reported a pain score of 30 or less ("mild pain while using stairs" or worse). Of these, 85\% (112 of 132) improved to "mild or occasional pain" or "no pain" over time, leaving $0.8 \%$ (20 of 2571) with some degree of persistent pain ("mild pain while using stairs" or worse). Of these 20 knees, 4 reported mild pain while walking and using stairs, 5 moderate occasional pain, 10 moderate continual and 1 severe at final follow-up.

The correlation between preoperative flexion and pain at 3, 5, or 7 year follow-up was $r=0.3206(p=0.0031)$. A percentage of $22.1 \%$ (563 of 2544) had preoperative flexion contracture greater than 5 degrees. Postoperatively, flexion contracture of 5 degrees occurred in 2.3\% (60 of 2571) of knees. Posterior cruciate excision occurred in $0.7 \%$ (17 of 2585 ) of knees.

There was a numerical, but not statistically significant, difference in pain scores between knees defined as having any instability (anteroposterior stability, $\mathrm{p}=0.8261$; mediolateral stability, $\mathrm{p}=0.4646$ ). Knees with any degree of anteroposterior instability (9 of 2585) reported an average pain score of $45.0( \pm 13.2)[10,50]$, compared to knees with no anteroposterior instability with average pain score of $47.7( \pm 6.7)$ [0, 50]. Knees with any degree of mediolateral instability (12 of 2585) gave an average postoperative pain score of $42.5( \pm 15.3)$ [10, 50], compared to those knees with no mediolateral instability with average postoperative pain score of $47.7( \pm 6.7)[0$, 50].

Postoperative pain scores from surgeries in different time periods were shown to be significantly different (difference 0.2 points of pain score out of $50, p=0.0102)$. In the 1990s the average pain score was $47.6( \pm 6.8$ ) [0. 50] and in the 2000s the average pain score was $47.4( \pm 7.5)[10,50]$. The $n=1564$ bilateral knees reported less pain, 0.6 points better pain score between 3 and 7 years, than unilateral knees with $p=0.0097$.

This analysis found that posterior cruciate ligament excision in a prosthesis designed for cruciate retention was associated with a 3.1-point decrease in the Knee Society pain score (more pain). It also found a 2.4-point decrease with a flexion contracture greater than 6 degrees, a 2.3-point decrease with flexion less than 105 degrees, and a 0.8-point increase with preoperative varus greater than 5 degrees. Compared to knees with a $85 \mathrm{~mm}$ tibial tray, knees with a $65 \mathrm{~mm}$ tray had 1.0 points less pain, knees with a $70 \mathrm{~mm}$ tray had 0.8 points less pain, and knees with a $80 \mathrm{~mm}$ tray had 0.3 points less pain (although this final comparison was not significant).

In the PKA cohort, there were 185 females (59.7\%) and 125 males (40.3\%) in the PKA. Average age was $65.7 \pm 9.8$ (range, 39 - 89 years), and average follow-up of $2.6 \pm 1.8$ years (range, $1.0-11.1$ ).

For the PKA, younger age was associated with greater pain $(-0.73$ pain score per decade of age, $p=0.0342$ (Table 2). Partial knee candidates were limited to those knees preoperatively with very good bone quality, lack of deformity, and anterior cruciate ligament intact. Other surgical and patient variables examined (with the numbers available) were: tibial size $(\mathrm{p}=0.8170)$, femoral size $(\mathrm{p}=0.7555)$, postoperative flexion $(\mathrm{p}=0.4204)$, postoperative flexion contracture $(\mathrm{p}=0.8859)$, postoperative varus alignment $(\mathrm{p}=0.3326)$, postoperative valgus alignment $(\mathrm{p}=0.2748)$, gender $(\mathrm{p}=0.3764)$, height $(\mathrm{p}=0.2532)$, weight $(\mathrm{p}=0.4474)$, and body mass index $(\mathrm{p}=$ $0.8430)$. 
Table 2. Factors associated with pain scores in UKA.

\begin{tabular}{ccc}
\hline Factor & Difference & p-value of factor \\
\hline Age (per decade) & -0.73 points & $\mathrm{p}=0.0077$ \\
\hline
\end{tabular}

\section{Discussion}

The present study found associations between pain after TKA and six patient factors between 2 and 8 years follow-up. We found a 3.1-point decrease (worse pain) in the Knee Society pain score among knees with an excised posterior cruciate ligament in a cruciate-retaining design, a 2.4-point decrease in knees with a postoperative flexion contracture greater than 6 degrees, and a 2.3-point decrease when knees had postoperative flexion less than 105 degrees. Osteonecrosis was another factor associated with postoperative pain. Smaller differences were found with preoperative varus greater than 5 degrees and small tibial components.

Research of factors in unexplained pain is both scarce and contradictory. Studies have shown "little consistent evidence" that patient characteristics influence pain and other variables analyzed in a recent systematic review of reports with greater than 500 cases [2]. Specifically, one study [7] examined in the review found that women have more pain after TKA, while another [8] did not find such a correlation. Other existing studies have examined the postoperative state of the posterior cruciate ligament [9]-[11], age [12], range of motion [13] [14], preoperative flexion contractures and hyperextension [6], psychological factors [15]-[17], and high-flexion [18] and fixed-bearing [19] prostheses, but as yet no consistent account of otherwise unexplained pain exists in the literature.

Our research uncovered significant associations of limited flexion and flexion contracture with worse pain scores. This finding agrees with data we had obtained previously [16] that found decreased pain scores in patients with a flexion contracture, and it coincides with discomfort found when stair-climbing and other ambulatory activities are hindered by inability to fully extend the knee joint. The difference of 2.4 points for knees with a flexion contracture greater than or equal to 6 degrees does not seem clinically significant, although we had found it to be statistically significant.

We found that knees with an excised posterior cruciate ligament had 3.1 points lower pain scores than knees without PCL excision. This both supports [11] and refutes [9] [10] previous research, reflecting the state of the literature on this issue. It is possible that confounding factors outside of PCL excision may have contributed to this finding. One study [11] cited selection bias in its finding that 17\% of TKAs with an excised PCL had a poor pain outcome. Potentially, knees that fared extremely poorly before arthroplasty would continue to exhibit lower scores post-arthroplasty and would never "catch up" with better-faring knees [20].

Psychological factors may have influenced all of the present study's findings, making the data limited in its application. Our center does not routinely record psychological states or attitudes toward surgery prior to and after total joint arthroplasty, so a retrospective study like this one would not be able to identify issues such as catastrophizing or unreasonable expectations that other studies have found [1] [14]-[16]. Further study is required if researchers are to understand the contributions of both physiological and psychological factors in knee pain.

One must keep in mind that these results only show correlations between pain and the six factors, not causation. The results of this study do not support the assertion that these six factors result in increased pain for the TKA patient; they can only say that the factors and increased pain were found together. Moreover, the differences shown in these results would likely not appear in a clinical evaluation, as the categories in the Knee Society pain score are separated by values of ten points. Regardless, the data suggest that further study, especially prospective analysis with a controlled patient population, might uncover more apparent and reliable effects of postoperative flexion, PCL excision, preoperative varus, and tibial size on pain scores.

There were notable differences with factors found to associate with pain between PKA and those with TKA. Unlike the TKA, younger ages tended to report more pain with the partial knee; while none of the factors affecting TKA pain were found to affect pain in the postoperative PKA. Although this may be entirely or partially due to patient selection of partial knee candidates, the mechanism of pain for PKA do appear (as we may have expected) to be quite different from that of TKA.

Pain following TKA appears to be affected by surgeon choices regarding the preservation of a posterior cruciate ligament and tibial tray size, preoperative variables such as anatomical alignment, and flexion. We found 
significant differences of greater than two points in the pain score in knees with a flexion contracture, posterior cruciate ligament excision, and suboptimal flexion, and we found significant differences of less than one point in knees with preoperative varus greater than 5 degrees and tibial trays of $65 \mathrm{~mm}$. These differences in pain might not be detectable in clinical follow-up, but the variables creating those different pain scores are worth further study.

\section{Conclusion}

The current study found six factors relevant to postoperative pain in TKA: postoperative flexion less than 105 degrees, flexion contracture more than 6 degrees, a posterior cruciate ligament excision, a smaller tibial tray size and diagnosis as osteonecrosis. It also found one factor, younger age, was associated with aggravating postoperative pain in partial knee arthroplasty. Surgeons should take these risk factors into consideration as we continue to understand pain after total and partial knee arthroplasty.

\section{Funding}

This study was an investigator-initiated study funded by the Joint Replacement Surgeons of Indiana (JRSI) foundation (Mooresville, IN). Funding played no role in the investigation, analysis, interpretation, or preparation of this manuscript.

\section{Conflict of Interests}

The authors declare no conflict of interests.

\section{References}

[1] Brander, V.A., Malhotra, S., Jet, J., Heinemann, A.W. and Stulberg, S.D. (1997) Outcome of Hip and Knee Arthroplasty in Persons Aged 80 Years and Older. Clinical Orthopedics Related Research, 345, 67-78. http://dx.doi.org/10.1097/00003086-199712000-00011

[2] Breugem, S.J., Sierevelt, I.N., Schafroth, M.U., et al. (2008) Less Anterior Knee Pain with a Mobile-Bearing Prosthesis Compared with a Fixed-Bearing Prosthesis. Clinical Orthopedics Related Research, 466, 1959-1965. http://dx.doi.org/10.1007/s11999-008-0320-6

[3] Brander, V.A., Stulberg, S.D., Adams, A.D., et al. (2003) Predicting Total Knee Replacement Pain: A Prospective, Observational Study. Clinical Orthopedics Related Research, 416, 27-36. http://dx.doi.org/10.1097/01.blo.0000092983.12414.e9

[4] Chaudhary, R., Beaupré, L.A. and Johnston, D.W. (2008) Knee Range of Motion during the First Two Years after Use of Posterior Cruciate-Stabilizing or Posterior Cruciate-Retaining Total Knee Prostheses. A Randomized Clinical Trial. The Journal of Bone and Joint Surgery (American), 90, 2579-2586. http://dx.doi.org/10.2106/JBJS.G.00995

[5] Elson, D.W. and Brenkel, I.J. (2006) Predicting Pain after Total Knee Arthroplasty. Journal of Arthroplasty, 21, 10471053. http://dx.doi.org/10.1016/j.arth.2005.12.010

[6] Forsythe, M.E., Dunbar, M.J., Hennigar, A.W., et al. (2008) Prospective Relation between Catastrophizing and Residual Pain Following Knee Arthroplasty: Two-Year Follow-Up. Pain Research \& Management, 13, 335-341.

[7] Gandhi, R., Davey, J.R. and Mahomed, N. (2009) Patient Expectations Predict Greater Pain Relief with Joint Arthroplasty. Journal of Arthroplasty, 24, 716-721. http://dx.doi.org/10.1016/j.arth.2008.05.016

[8] Hawker, G., Wright, J., Coyte, P., et al. (1998) Health-Related Quality of Life after Knee Replacement. The Journal of Bone and Joint Surgery (American), 80, 163-173.

[9] Kim, J., Nelson, C.L. and Lotke, P.A. (2004) Stiffness after Total Knee Arthroplasty. Prevalence of the Complication and Outcomes of Revision. The Journal of Bone and Joint Surgery (American), 86, 1479-1484.

[10] Lavernia, C., D’Apuzzo, M., Rossi, M.D. and Lee, D. (2009) Is Postoperative Function After Hip or Knee Arthroplasty Influenced by Preoperative Functional Levels? Journal of Arthroplasty, 24, 1033-1043. http://dx.doi.org/10.1016/j.arth.2008.09.010

[11] Lingard, E.A., Katz, J.N., Wright, E.A. and Sledge, C.B., Kinemax Outcomes Group (2004) Predicting the Outcome of Total Knee Arthroplasty. The Journal of Bone and Joint Surgery (American), 86, 2179-2186.

[12] Mont, M.A., Serna, F.K., Krackow, K.A. and Hungerford, D.S. (1996) Exploration of Radiographically Normal Total Knee Replacements for Unexplained Pain. Clinical Orthopedics Related Research, 331, 216-220. 
http://dx.doi.org/10.1097/00003086-199610000-00030

[13] Noble, P.C., Conditt, M.A., Cook, K.F. and Mathis, K.B. (2006) The John Insall Award: Patient Expectations Affect Satisfaction with Total Knee Arthroplasty. Clinical Orthopedics Related Research, 452, 35-43. http://dx.doi.org/10.1097/01.blo.0000238825.63648.1e

[14] Ritter, M.A. and Campbell, E.D. (1987) Effect of Range of Motion on the Success of a Total Knee Arthroplasty. Journal of Arthroplasty, 2, 95-97. http://dx.doi.org/10.1016/S0883-5403(87)80015-3

[15] Ritter, M.A., Harty, L.D., Davis, K.E., Meding, J.B. and Berend, M.E. (2003) Predicting Range of Motion after Total Knee Arthroplasty. Clustering, Log-Linear Regression, and Regression Tree Analysis. The Journal of Bone and Joint Surgery (American), 85, 1278-1285.

[16] Ritter, M.A., Lutgring, J.D., Davis, K.E., Berend, M.E., Pierson, J.L. and Meneghini, R.M. (2007) The Role of Flexion Contracture on Outcomes in Primary Total Knee Arthroplasty. Journal of Arthroplasty, 22, 1092-1096. http://dx.doi.org/10.1016/j.arth.2006.11.009

[17] Ritter, M.A., Thong, A.E., Davis, K.E., Berend, M.E., Meding, J.B. and Faris, P.M. (2004) Long-Term Deterioration of Joint Evaluation Scores. The Journal of Bone and Joint Surgery (British), 86, 438-442. http://dx.doi.org/10.1302/0301-620X.86B3.14243

[18] Santaguida, P.L., Hawker, G.A., Hudak, P.L., Glazier, R., Mahomed, N.N., Kreder, H.J., et al. (2008) Patient Characteristics Affecting the Prognosis of Total Hip and Knee Joint Arthroplasty: A Systematic Review. Canadian Journal of Surgery, 51, 428-436.

[19] Seon, J.K., Park, S.J., Lee, K.B., Yoon, T.R., Kozanek, M. and Song, E.K. (2009) Range of Motion in Total Knee Arthroplasty: A Prospective Comparison of High-Flexion and Standard Cruciate-Retaining Designs. The Journal of Bone and Joint Surgery (American), 91, 672-679. http://dx.doi.org/10.2106/jbjs.h.00300

[20] Whiteside, L.A. (1991) The Effect of Patient Age, Gender, and Tibial Component Fixation on Pain Relief after Cementless Total Knee Arthroplasty. Clinical Orthopedics Related Research, 271, 21-27.

http://dx.doi.org/10.1097/00003086-199110000-00005 